\title{
Association of extracerebral organ failure with 1-year survival and healthcare- associated costs after cardiac arrest: an observational database study
}

Pirkka T. Pekkarinen ${ }^{1 *}$ D, Minna Bäcklund ${ }^{1}$, Ilmar Efendijev ${ }^{1}$, Rahul Raj ${ }^{2}$, Daniel Folger ${ }^{1}$, Erik Litonius ${ }^{1}$, Ruut Laitio ${ }^{3}$, Stepani Bendel ${ }^{4}$, Sanna Hoppu ${ }^{5}$, Tero Ala-Kokko ${ }^{6,7}$, Matti Reinikainen ${ }^{8}$ and Markus B. Skrifvars ${ }^{9}$

\begin{abstract}
Background: Organ dysfunction is common after cardiac arrest and associated with worse short-term outcome, but its impact on long-term outcome and treatment costs is unknown.

Methods: We used nationwide registry data from the intensive care units (ICU) of the five Finnish university hospitals to evaluate the association of 24-h extracerebral Sequential Organ Failure Assessment (24h-EC-SOFA) score with 1-year survival and healthcare-associated costs after cardiac arrest. We included adult cardiac arrest patients treated in the participating ICUs between January 1, 2003, and December 31, 2013. We acquired the confirmed date of death from the Finnish Population Register Centre database and gross 1-year healthcare-associated costs from the hospital billing records and the database of the Finnish Social Insurance Institution.

Results: A total of 5814 patients were included in the study, and 2401 were alive 1 year after cardiac arrest. Median (interquartile range (IQR)) 24h-EC-SOFA score was 6 (5-8) in 1-year survivors and 7 (5-10) in non-survivors. In multivariate regression analysis, adjusting for age and prior independency in self-care, the 24h-EC-SOFA score had an odds ratio (OR) of 1.16 (95\% confidence interval (Cl) 1.14-1.18) per point for 1-year mortality.

Median (IQR) healthcare-associated costs in the year after cardiac arrest were $€ 47,000$ ( $€ 28,000-75,000)$ in 1-year survivors and $€ 12,000$ (€6600-25,000) in non-survivors. In a multivariate linear regression model adjusting for age and prior independency in self-care, an increase of one point in the 24h-EC-SOFA score was associated with an increase of $€ 170$ (95\% Cl €150-190) in the cost per day alive in the year after cardiac arrest. In the same model, an increase of one point in the 24h-EC-SOFA score was associated with an increase of $€ 4400$ (95\% Cl €3300-5500) in the total healthcare-associated costs in 1-year survivors.
\end{abstract}

Conclusions: Extracerebral organ dysfunction is associated with long-term outcome and gross healthcareassociated costs of ICU-treated cardiac arrest patients. It should be considered when assessing interventions to improve outcomes and optimize the use of resources in these patients.

Keywords: Cardiac arrest, OHCA, IHCA, Cost of care, Cost-effectiveness, Organ failure, Multiple organ failure, Outcome, Post cardiac arrest syndrome, SOFA

\footnotetext{
* Correspondence: pirkka.pekkarinen@helsinkifi

${ }^{1}$ Division of Intensive Care Medicine, Department of Anaesthesiology,

Intensive Care and Pain Medicine, University of Helsinki and Helsinki

University Hospital, PB 340, 00029 Helsinki, HUS, Finland

Full list of author information is available at the end of the article
}

(c) The Author(s). 2019 Open Access This article is distributed under the terms of the Creative Commons Attribution 4.0 International License (http://creativecommons.org/licenses/by/4.0/), which permits unrestricted use, distribution, and reproduction in any medium, provided you give appropriate credit to the original author(s) and the source, provide a link to the Creative Commons license, and indicate if changes were made. The Creative Commons Public Domain Dedication waiver (http://creativecommons.org/publicdomain/zero/1.0/) applies to the data made available in this article, unless otherwise stated. 


\section{Background}

Cardiac arrest is an extensively studied topic due to its devastating consequences [1]. Cardiac arrest may itself cause end-organ damage leading to life-threatening organ dysfunction despite successful return of spontaneous circulation (ROSC) [2]. In addition to this the systemic inflammatory response which follows (often called "post cardiac arrest syndrome") is characterized by a sepsis-like inflammatory response which may precipitate multiple organ failure (MOF) and death $[3,4]$. Contemporary treatment of cardiac arrest patients in the ICU is focused on supporting the recovery of the central nervous system and the myocardium after ischaemia. The dysfunction of other organ systems after cardiac arrest may have an impact on recovery but has received less attention.

The Sequential (or sepsis-related) Organ Failure Assessment (SOFA) score is widely used for estimating the severity of MOF. The SOFA score was originally developed for the evaluation of organ failure in sepsis [5], but it has later been validated as a general scoring system for all critically ill patients [6-11].

The extracerebral (EC-)SOFA score [i.e. SOFA score excluding the central nervous system (CNS) sub-score] can be used to evaluate the degree of MOF outside the central nervous system. Two previous single-centre studies have shown an association of EC-SOFA with in-hospital and 28-day mortality in cardiac arrest patients [12, 13], and one multi-centre study found an association of admission renal SOFA sub-score with ICU mortality [14].

The aim of this study was to quantify the impact of early extracerebral organ failure, measured as the EC-SOFA score at $24 \mathrm{~h}$ (24h-EC-SOFA), on 1-year survival and healthcare-associated costs in cardiac arrest patients treated in the ICU.

\section{Methods}

\section{Study population}

This is a retrospective study conducted at the five university hospitals of Finland. These five centres serve a population of 3.2 million covering $60 \%$ of the entire population of Finland. We included adult cardiac arrest patients treated in the participating ICUs between January 1, 2003, and December 31, 2013. The year 2003 was chosen as the starting point because it was the first year when therapeutic hypothermia became widely used in the treatment of cardiac arrest patients in the participating centres [15]. Only the first event of cardiac arrest was considered. Cardiac arrest was recognized as admission diagnosis of cardiac arrest according to Acute Physiology and Chronic Health Evaluation III (APACHE III) or positive value for Therapeutic Intervention Scoring System 76 (TISS-76) item of "cardiac arrest and/or countershock within past $48 \mathrm{~h}$ ”. Complete data for admission date and day of death/vital status at the end of 1-year follow-up was required for inclusion. Patients with missing SOFA score were excluded.

\section{Database}

The Finnish social security and healthcare system is government-based. Each Finnish resident is assigned a unique personal identification code which is used to identify the person in medical records, social security records, and national registers. We used this identification code to combine data from different sources.

Medical data were extracted from the Finnish Intensive Care Consortium (FICC) database [16]. These included the SOFA score and the premorbid physical status as a simplified World Health Organization-Eastern Cooperative Oncology Group (WHO/ECOG) classification [17], where "independent" was defined as the patient being independent in self-care and "dependent" was defined as the patient being partly or fully dependent on help in self-care prior to hospital admission. We linked these data with the confirmed date of death obtained from the Finnish Population Register Centre database, which records all deaths of Finnish residents.

Furthermore, we linked data on gross 1-year healthcare-associated costs from the hospital billing records, rehabilitation costs and social security costs obtained from the Social Insurance Institution. The hospital costs include all costs until hospital discharge (diagnostics, ICU stay, ward stay, operative treatment etc.). The rehabilitation costs were calculated by multiplying the number of days spent in the rehabilitation unit with the average price per day for units of the corresponding level. The Finnish Social Insurance Institution reimburses disability and sickness allowances, private physician and physiotherapist expenses, prescription drug expenses and medical transportation expenses. All social insurance reimbursements during the year following hospital admission were included. Effective cost per survivor (ECPS) was calculated by dividing the sum of total costs for all patients with the number of survivors [18]. We adjusted all costs to the value of euro in the year 2013 according to the consumer price index (CPI) in Finland.

\section{Nested cohort}

We performed an additional analysis of a nested cohort consisting of patients treated in one (Helsinki University Hospital) of the five centres of the full data. For this cohort, additional data were available according to the Utstein criteria $[19,20]$ including the delay from collapse to return of spontaneous circulation (ROSC), type of initial rhythm during cardiac arrest (shockable or not), whether the cardiac arrest was witnessed or not, and 1-year cerebral performance category (CPC) [21, 22]. 
We defined good neurologic outcome as 1-year CPC 1 or 2 and poor neurologic outcome as 1-year CPC 3 to 5 . Furthermore, the location where cardiac arrest had occurred was recorded, allowing stratification of the nested cohort data in out-of-hospital cardiac arrest (OHCA) and in-hospital cardiac arrest (IHCA). Cardiac arrests in the ICU (ICUCA) were included in the IHCA group in this study.

\section{Statistics}

We performed the statistical analyses with the SPSS software (version 24.0, IBM, Armonk, NY, USA). We present continuous data as medians with interquartile ranges (IQR) and categorical data as percentages of the whole. We tested group differences with a chi-square test, Student's $t$ test, Mann-Whitney $U$ test or Kruskal-Wallis test, as appropriate. We performed Kaplan-Meier analysis to evaluate the difference in 1-year survival across quartiles of the 24h-EC-SOFA score. We used logistic regression models to search for variables that were independently associated with outcome. For testing the association of the 24h-EC-SOFA score with healthcare-associated costs, we used linear regression models. To avoid the confounding effect of early deaths causing low costs, we calculated a "cost per day alive" variable by dividing the total costs of a given patient by the number of days the patient remained alive during the first year after the cardiac arrest. If the patient was still alive 1 year after the arrest, then the cost per day alive variable was calculated by dividing the total costs during the year after cardiac arrest by 365 .

\section{STROBE statement}

The manuscript was completed according to STROBE standards [23].

\section{Results}

In total, 5814 patients were included in the study, and of these, 2401 were alive 1 year after the cardiac arrest. Median (IQR) survival time for 1-year non-survivors was 5 (2-14) days. Patient selection is shown in Fig. 1. Characteristics of the study population are described in Table 1, and those of the nested cohort in Additional file 1: Table S1. The values of the $24 \mathrm{~h}$-EC-SOFA score were normally distributed in the groups stratified by outcome, with a range from 0 to 18 (Additional file 2: Figure S1). The distribution of the full 24h-SOFA score ranged from 0 to 21 (Additional file 2: Figure S1).

\section{Outcome}

In Kaplan-Meier analysis, 1-year survival was significantly different between quartiles of 24h-EC-SOFA (logrank $P<0.001$; Fig. 2). One-year survival had a rising trend from 38\% in 2003 to $47 \%$ in 2013 (Additional file 3: Figure S2). In a logistic regression model adjusting for age and the premorbid physical status, the 24h-EC-SOFA score was associated with 1-year mortality [odds ratio (OR) 1.16 and $95 \%$ confidence interval (CI) 1.14-1.18; Table 2]. Similarly, the full 24h-SOFA score was associated with 1-year mortality in a logistic regression model adjusting for age and the premorbid physical status (OR 1.22, 95\% CI 1.20-1.24; Additional file 4: Table S2). All sub-scores, except the cardiovascular sub-score, were independently associated with 1-year mortality when the individual EC-sub-scores were tested (respiration, OR 1.18, 95\% CI 1.12-1.25; coagulation, OR 1.09, 95\% CI 1.00-1.18; liver, OR 1.23, 95\% CI 1.10-1.37; renal, OR 1.52, 95\% CI 1.441.61; Additional file 5: Table S3). Univariate analysis of the variables used in the logistic regression models is presented in Additional file 6: Table S4.

\section{Healthcare-associated costs}

The sum of costs recorded in all 5814 patients during the year following cardiac arrest was $€ 230,000,000$. The 1-year ECPS was $€ 96,000$. Median costs in the first year after cardiac arrest were $€ 47,000$ in 1-year survivors and $€ 12,000$ in non-survivors (Table 1). During the study years, ECPS remained between $€ 85,000$ and $€ 110,000$ (Additional file 7: Figure S3). Median costs recorded for 1-year survivors were lowest in $2003(€ 38,000)$ and highest in 2004 ( $€ 52,000)$, plateauing thereafter (Additional file 8: Figure S4). Median costs recorded for 1-year non-survivors had a rising trend from $€ 9900$ in 2003 to $€ 16,000$ in 2013(Additional file 8: Figure S4). When the data were divided in quartiles of the 24h-EC-SOFA score, the total costs of 1-year survivors differed significantly between groups $(P<0.001)$; higher 24h-EC-SOFA score was associated with higher costs (Fig. 3). The same was true when the quartiles of the 24h-EC-SOFA score were tested for the association with cost per day alive during the first year after cardiac arrest $(P<0.001)$. The differences were similar for quartiles of the $24 \mathrm{~h}$-SOFA score $(P<0.001$ for the difference in total costs in 1-year survivors and $P<0.001$ for the difference in cost per day alive; Fig. 3).

In a linear regression model adjusting for age and premorbid physical status, an increase of one point in the 24h-EC-SOFA score was associated with an increase of $€ 170$ (95\% CI €150-190) in the cost per day alive in the first year after cardiac arrest (Table 3). When the EC-sub-scores were tested, renal, cardiovascular, respiratory and coagulation sub-scores were significantly associated with an increase in the cost per day alive (increase per one point rise: respiration, $€ 120$, 95\% CI €61 - 170; coagulation, €300, 95\% CI €230 370; cardiovascular, €90, 95\% CI €41 - 140; renal, €400, 95\% CI $€ 350$ - 440; Additional file 9: Table S5). In 1-year survivors, an increase of one point in the 24h-EC-SOFA score was associated with an increase of $€ 4400$ (95\% CI €33005500) in total healthcare-associated costs recorded during the year following the cardiac arrest (Table 4). When the EC-sub-scores were tested, renal, respiratory and coagulation 


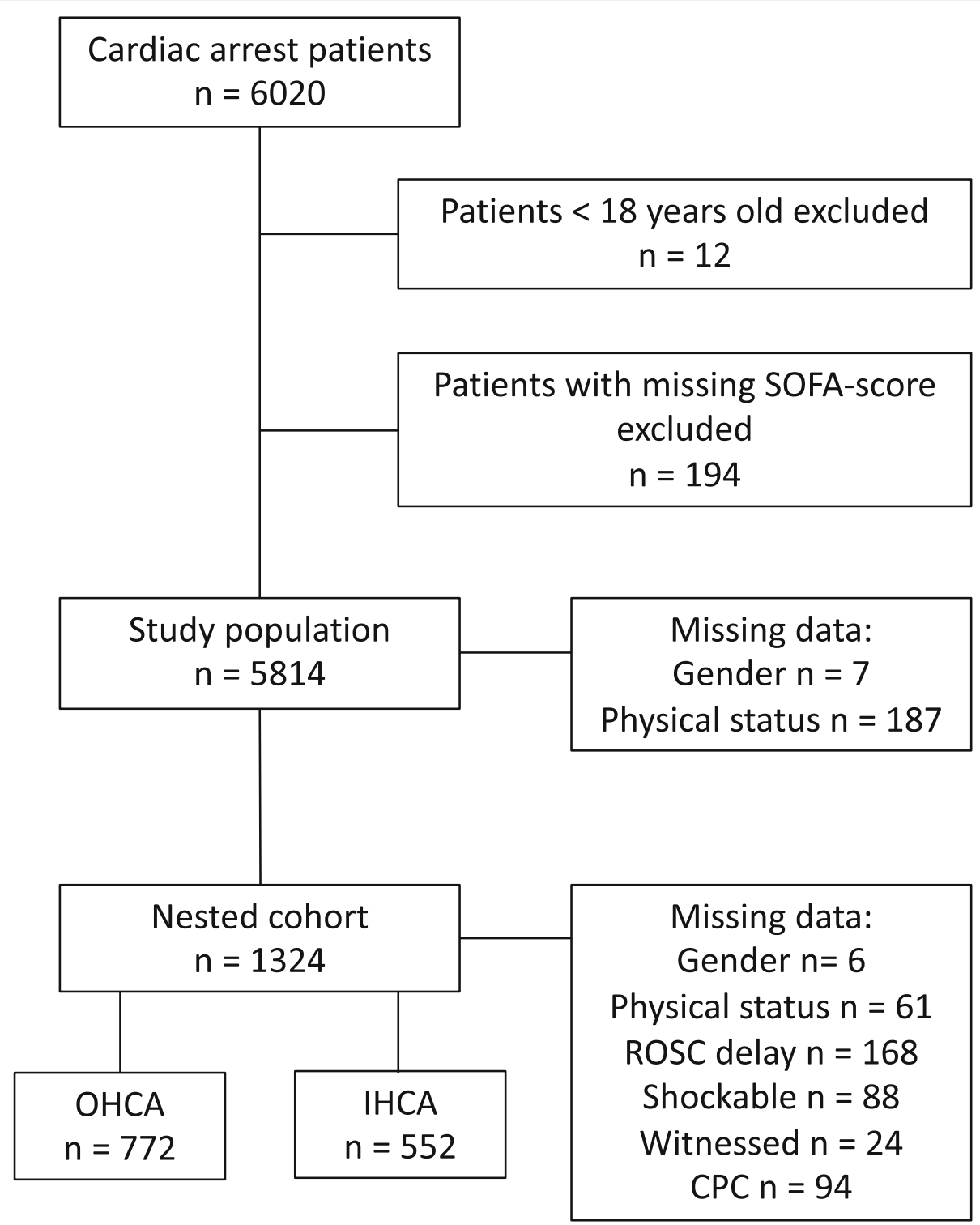

Fig. 1 Flow chart of patient selection

Table 1 Characteristics of the study population

\begin{tabular}{lllll}
\hline & All & One-year survivors & One-year non-survivors \\
$N=3413(59 \%)$ & $P$ value \\
\hline Age (years) & $N=5814(100 \%)$ & $N=2401(41 \%)$ & $67(57-76)$ & $<0.01$ \\
Gender (male) & $65(56-74)$ & $63(54-71)$ & $69 \%$ & $<0.01$ \\
Physical status (independent) & $71 \%$ & $72 \%$ & $79 \%$ & $<0.01$ \\
24 h-EC-SOFA & $84 \%$ & $92 \%$ & $15-10)$ & $<0.01$ \\
24 h-SOFA & $7(5-9)$ & $8(6-10)$ & $12(6.6-25)$ & $<0.01$ \\
One-year costs $(€ 1000)$ & $9(7-12)$ & $47(28-75)$ & $2200(1300-3400)$ & $<0.01$ \\
Cost per day alive $(€)$ & $23(10-50)$ & $130(77-200)$ & & $<0.01$ \\
\hline
\end{tabular}

${ }^{1} P$ value for comparison between outcome groups

${ }^{2}$ Simplified WHO/ECOG-classification before cardiac arrest 


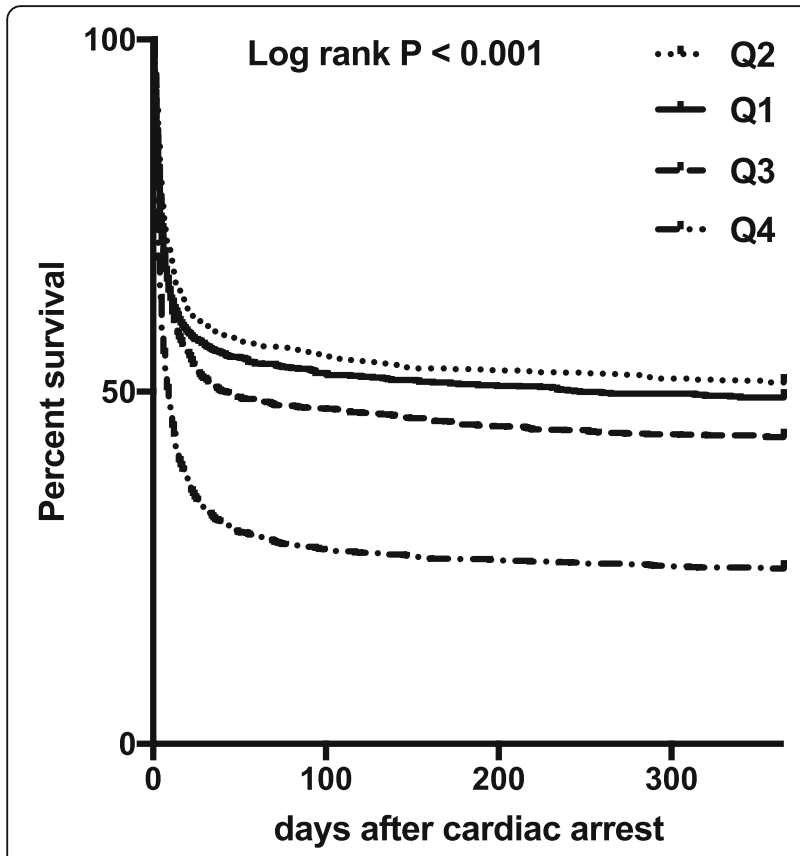

Fig. 2 Kaplan-Meier curves for survival up to 1 year after cardiac arrest in quartiles of the EC-SOFA score. Q1 quartile 1, EC-SOFA score 0-4; Q2, 5-6; Q3, 7-8; Q4, 9-18. Logrank P value for equality of survival distributions across quartiles of EC-SOFA

sub-scores were significantly associated with an increase of total healthcare-associated costs recorded during the year following the cardiac arrest (increase per one point rise: respiration, €7300, 95\% CI €4800 - 9800; coagulation, €4600, 95\% CI €1000 - 8300; renal, €7900, 95\% CI €5000 - 11,000; Additional file 10: Table S6).

\section{Outcome in the nested cohort}

For the nested cohort, the logistic regression model was complemented with variables describing conditions during resuscitation: whether the initial cardiac rhythm during cardiac arrest was shockable, the delay from collapse to ROSC and whether the cardiac arrest was witnessed. In this model, the 24h-EC-SOFA score was associated with 1-year mortality (OR 1.19, 95\% CI 1.12-1.25 per point of 24h-EC-SOFA) and poor neurologic outcome (OR 1.15, 95\% CI 1.09-1.21 per point of 24h-EC-SOFA; Table 2). When the individual EC-sub-scores were tested, respiratory and renal sub-scores were independently associated with both 1-year mortality (respiration, OR 1.16, 95\% CI 1.02-1.32; renal, OR 1.50, 95\% CI $1.32-1.70$ ) and poor neurologic outcome (respiration, OR 1.17, 95\% CI 1.02-1.33; renal, OR 1.42, 95\% CI 1.25-1.63; Additional file 11: Table S7). The association of the 24h-EC-SOFA score with 1-year mortality and poor neurologic outcome remained significant in subgroup analysis of both OHCA (1-year mortality OR 1.20, 95\% CI 1.10-1.31 per point of 24h-EC-SOFA; poor neurologic outcome OR $1.15,95 \%$ CI $1.05-1.25$ per point of 24h-EC-SOFA) and IHCA patients (1-year mortality OR $1.15,95 \%$ CI $1.06-1.24$ per point of $24 \mathrm{~h}$-EC-SOFA; poor neurologic outcome OR 1.11, 95\% CI 1.03-1.21 per point of 24h-EC-SOFA; Additional file 12: Table S8).

\section{Healthcare-associated costs in the nested cohort}

In the nested cohort, the total costs documented for the 1324 patients was $€ 65,000,000$ and 1-year ECPS was $€ 94,000$. The cohort included $772 \mathrm{OHCA}$ and 552 IHCA patients. Median (IQR) survival time for 1-year non-survivors was 5 (3-10) days in OHCA and 8 (3-20) days in IHCA. In the nested cohort, the linear regression model adjusting for age and premorbid physical status indicated an association of an increase of one point in the 24h-EC-SOFA score with a €280 (95\% CI €230-330) increase in the cost per day alive. For 1-year survivors, the increase in the total healthcare-associated costs in the year after cardiac arrest was $€ 7700$ (95\% CI $€ 5700$ 9700) per one-point rise in the 24h-EC-SOFA score. When the model was complemented with Utstein

Table 2 Logistic regression model of the association of the 24h-EC-SOFA score with 1-year outcome

\begin{tabular}{|c|c|c|c|c|c|c|c|c|c|}
\hline & \multirow{2}{*}{\multicolumn{3}{|c|}{$\begin{array}{l}\text { Full data } \\
\text { One-year mortality }\end{array}$}} & \multicolumn{6}{|c|}{ Nested cohort } \\
\hline & & & & \multicolumn{3}{|c|}{ One-year mortality } & \multicolumn{3}{|c|}{ Poor neurologic outcome $^{1}$} \\
\hline & $\overline{O R}$ & $95 \% \mathrm{Cl}$ & $P$ & $\overline{O R}$ & $95 \% \mathrm{Cl}$ & $P$ & $\overline{\mathrm{OR}}$ & $95 \% \mathrm{Cl}$ & $P$ \\
\hline Age (year) & 1.02 & $1.01-1.02$ & $<0.01$ & 1.03 & $1.02-1.04$ & $<0.01$ & 1.03 & $1.02-1.04$ & $<0.01$ \\
\hline Physical status (dependent) ${ }^{2}$ & 2.60 & $2.18-3.09$ & $<0.01$ & 2.76 & $1.60-4.74$ & $<0.01$ & 3.72 & $2.01-6.88$ & $<0.01$ \\
\hline Not shockable ${ }^{3}$ & & & & 3.55 & $2.61-4.82$ & $<0.01$ & 3.89 & $2.82-5.37$ & $<0.01$ \\
\hline ROSC delay $(\mathrm{min})^{4}$ & & & & 1.05 & $1.03-1.06$ & $<0.01$ & 1.05 & $1.03-1.06$ & $<0.01$ \\
\hline Not witnessed ${ }^{5}$ & & & & 1.87 & $1.19-2.93$ & $<0.01$ & 2.05 & $1.26-3.32$ & $<0.01$ \\
\hline 24h-EC-SOFA (point) & 1.16 & $1.14-1.18$ & $<0.01$ & 1.19 & $1.12-1.25$ & $<0.01$ & 1.15 & $1.09-1.21$ & $<0.01$ \\
\hline
\end{tabular}

${ }^{1}$ Cerebral performance category (CPC) 3-5 1 year after cardiac arrest

${ }^{2}$ Simplified WHO/ECOG-classification before cardiac arrest

${ }^{3}$ Not shockable, initial cardiac rhythm during resuscitation not shockable (asystole/pulseless electrical activity)

${ }^{4} \mathrm{ROSC}$ delay, time from collapse to return of spontaneous circulation

${ }^{5}$ Not witnessed, collapse not witnessed 
Total costs in one-year survivors
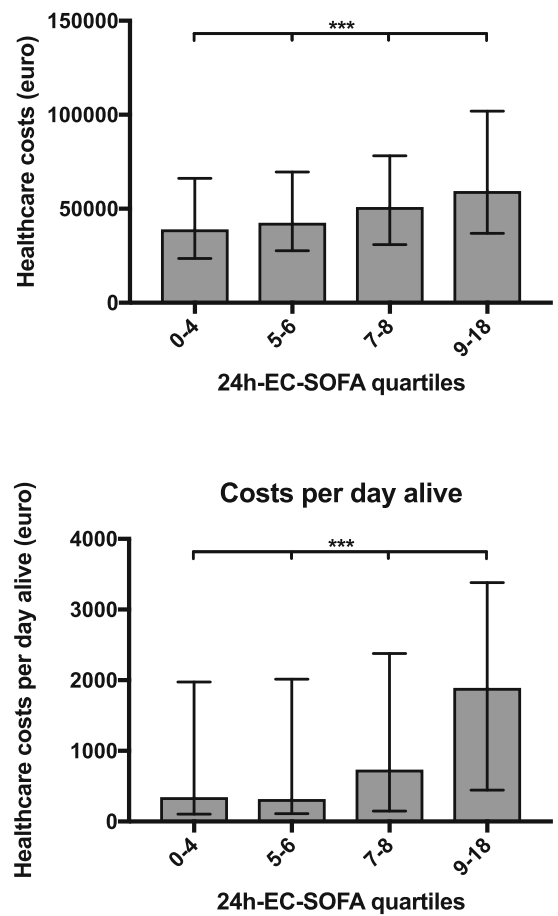

Total costs in one-year survivors
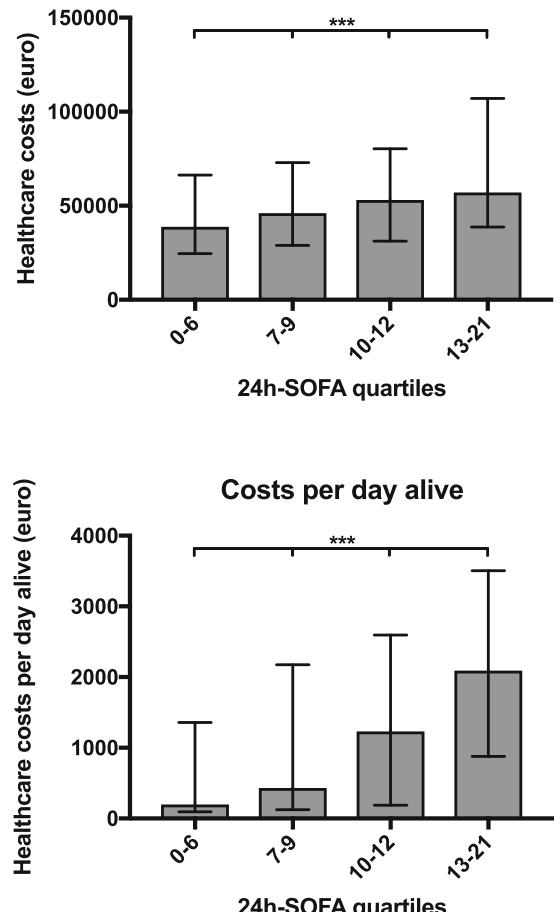

Fig. 3 Healthcare-associated costs recorded during the first year after cardiac arrest in quartiles of 24h-EC-SOFA and 24h-SOFA scores. Upper panels: Total healthcare-associated costs in 1-year survivors in quartiles of 24h-EC-SOFA score (left) and full 24h-SOFA score including CNS subscore (right). Lower panels: healthcare-associated costs per day alive in quartiles of the 24h-EC-SOFA score (left) and full 24h-SOFA score including CNS sub-score (right). The cost per day alive was calculated by dividing the total cost by the number of days from cardiac arrest to death. For 1year survivors, the cost per day alive was calculated by dividing the total cost by 365 days. Median values (bars) with interquartile range (whiskers) are presented. ${ }^{* *} P<0.001$ for the difference between the four groups (Kruskal-Wallis test)

variables, the increase in the cost per day alive was $€ 210$ (95\% CI $€ 170-260$ ) per 24h-EC-SOFA point. In 1-year survivors, total healthcare-associated costs increased $€ 7200$ (95\% CI $€ 5000-9400$ ) per each additional 24h-EC-SOFA point (Tables 3 and 4). When the data were stratified according to cardiac arrest location, the increase in the cost per day alive in OHCA was $€ 210$ (95\% CI €150-280) and in IHCA $€ 200$ (95\% CI €120-270) per 24h-EC-SOFA point. In OHCA patients, the total healthcare-associated costs in 1-year survivors increased $€ 2500$ (95\% CI $€ 200-4800)$ and in IHCA patients $€ 11,000$ (95\% CI

Table 3 Linear model of the association of the 24h-EC-SOFA score with 1-year healthcare-associated costs per day alive

\begin{tabular}{|c|c|c|c|c|c|c|}
\hline & \multicolumn{6}{|c|}{ Cost per day alive $(€)$} \\
\hline & \multicolumn{3}{|c|}{ Full data } & \multicolumn{3}{|c|}{ Nested cohort } \\
\hline & $B$ & $95 \% \mathrm{Cl}$ & $P$ & $B$ & $95 \% \mathrm{Cl}$ & $P$ \\
\hline Age (year) & -3.8 & $-8.4-0.80$ & NS & 3.8 & $-5.1-13$ & NS \\
\hline Physical status (dependent) $^{1}$ & 110 & $-67-284$ & NS & 220 & $-240-680$ & NS \\
\hline Not shockable ${ }^{2}$ & & & & 780 & $510-1,100$ & $<0.01$ \\
\hline $\operatorname{ROSC}(\min )^{3}$ & & & & 37 & $25-49$ & $<0.01$ \\
\hline Not witnessed ${ }^{4}$ & & & & 200 & $-200-610$ & NS \\
\hline 24h-EC-SOFA (point) & 170 & $150-190$ & $<0.01$ & 210 & $170-260$ & $<0.01$ \\
\hline
\end{tabular}

NS not statistically significant $(P>0.05)$

${ }^{1}$ Simplified WHO/ECOG-classification before cardiac arrest

${ }^{2}$ Not shockable, initial cardiac rhythm during resuscitation not shockable (asystole/pulseless electrical activity)

${ }^{3} \mathrm{ROSC}$ delay, time from collapse to return of spontaneous circulation

${ }^{4}$ Not witnessed, collapse not witnessed 
Table 4 Linear model of the association of the 24h-EC-SOFA score with 1-year total healthcare-associated costs (per €1000) in 1-year survivors

\begin{tabular}{|c|c|c|c|c|c|c|}
\hline & \multicolumn{6}{|c|}{ Total costs in 1-year survivors (€1000) } \\
\hline & \multicolumn{3}{|c|}{ Full data } & \multicolumn{3}{|c|}{ Nested cohort } \\
\hline & $\bar{B}$ & $95 \% \mathrm{Cl}$ & $P$ & $\bar{B}$ & $95 \% \mathrm{Cl}$ & $P$ \\
\hline Age (year) & -0.85 & $-1.1--0.64$ & $<0.01$ & -0.48 & $-0.85--0.10$ & 0.01 \\
\hline Physical status (dependent) $)^{1}$ & 15 & $5.1-25$ & $<0.01$ & 28 & $2.4-54$ & 0.03 \\
\hline Not shockable ${ }^{2}$ & & & & 15 & $3.2-27$ & 0.01 \\
\hline $\operatorname{ROSC}(\min )^{3}$ & & & & 0.047 & $-0.45-0.55$ & NS \\
\hline Not witnessed ${ }^{4}$ & & & & -20 & $-39--0.91$ & 0.04 \\
\hline 24h-EC-SOFA (point) & 4.4 & $3.3-5.5$ & $<0.01$ & 7.2 & $5.0-9.4$ & $<0.0$ \\
\hline
\end{tabular}

NS not statistically significant $(P>0.05)$

${ }^{1}$ Simplified WHO/ECOG-classification before cardiac arrest

${ }^{2}$ Not shockable, initial cardiac rhythm during resuscitation not shockable (asystole/pulseless electrical activity)

${ }^{3} \mathrm{ROSC}$ delay, time from collapse to return of spontaneous circulation

${ }^{4}$ Not witnessed, collapse not witnessed

$€ 5600-16,000)$ per each additional 24h-EC-SOFA point (Additional files 13 and 14: Tables S9 and S10).

\section{Discussion}

In this large multi-centre cohort, we show that extracerebral organ failure is an important contributor to both long-term outcome and increased healthcare-associated costs in cardiac arrest patients. Importantly, we measured extracerebral organ failure with a simple method, the 24h-EC-SOFA, and were able to quantify its association with long-term resource use in cardiac arrest survivors.

\section{Outcome and SOFA}

Previous studies have shown an association of admission SOFA and EC-SOFA with 28-/30-day mortality in single-centre settings of OHCA patients [13, 24, 25]. Association with neurologic outcome has produced conflicting results $[24,25]$. In a single-centre material including both OHCA and IHCA patients, the highest EC-SOFA score during the first $72 \mathrm{~h}$ of ICU stay was associated with in-hospital mortality and cardiovascular and respiratory sub-scores were independently predictive of in-hospital mortality [12]. In a multi-centre database study including both OHCA and IHCA patients, ICU non-survivors had higher cardiovascular, respiratory and renal SOFA sub-scores upon ICU admission but only renal sub-score was independently associated with ICU-mortality [14].

To the best of our knowledge, our current study is the largest study on the association of EC-SOFA with cardiac arrest patient outcome published to date. In our multi-centre setting with a mixed cohort of OHCA and ICHA patients, the association of EC-SOFA with 1-year outcome was clear. In contrast to other sub-scores, the cardiovascular sub-score was not independently associated with the outcome in our cohort, since almost three quarters of patients had 24-h cardiovascular sub-score of 3 or 4 , regardless of the outcome group. In line with previous studies, respiratory and renal sub-scores had a clear association with the outcome in our study population.

It is important to notice that the large overlap between the outcome groups does not allow the use of 24h-EC-SOFA for predicting outcome on a single patient level. However, our results emphasize the importance of the evolving multiple organ failure for long-term morbidity, mortality and healthcare resource use in cardiac arrest patients on population level. This association could justify the use of SOFA or EC-SOFA score as an end-point in small randomized controlled trials on cardiac arrest in the future instead of mortality, for which the studies often prove underpowered.

\section{Healthcare-associated costs}

Recently, there has been a growing interest in the obvious socioeconomic burden of cardiac arrest [26]. One of the first studies assessing the healthcare resource use in cardiac arrest reported combined pre- and in-hospital costs for OHCA 6-month survivors to be $€ 36,000$ (expressed as 2013 euros) [27]. More recent studies have reported in-hospital costs of $€ 50,000-60,000$ in 2013 euros for patients surviving to hospital discharge after OHCA or IHCA [28-30]. One previous study reported OHCA survivors to have combined pre-, in- and post-hospital healthcare-associated costs of $€ 37,000$ in 2013 euros in a 6-month follow-up [31]. In a recent study of single-centre data published by our group, combined in- and post-hospital healthcare costs in 1-year follow-up were $€ 59,000$ for OHCA and $€ 84,000$ for IHCA hospital survivors in 2013 euros [18].

In our current study, the association of 24h-EC-SOFA with healthcare-associated costs was clear. Most of the 
sub-scores (renal and respiratory sub-scores in particular) had also independent association with costs. This suggests that early organ failure after cardiac arrest leads to both short- and long-term morbidity reflected as increased inand post-hospital expenses.

The analysis of our nested cohort clearly shows that the association of organ failure with outcome and costs is not redundant to more detailed information considering the conditions during and after cardiac arrest and resuscitation. The similarity of the ECPS values of the nested cohort and the full data ( $€ 94,000$ and $€ 96,000$, respectively) suggest that the case-mix in the nested cohort was not markedly different from that of the full data.

\section{Strengths and limitations of the study}

This study has a number of strengths. It is a large multicentre study conducted in a country of governmentfunded social security and healthcare systems. The follow-up time of 1 year is long enough to reliably dissect long-term survivors from non-survivors. We have exploited the unique possibility provided by the nature of the government-funded system to combine data from different registries to acquire information on long-term survival and gross healthcare-associated costs from different healthcare providers. Naturally, there are also limitations. Our data are limited to one country-the generalizability to other healthcare systems is not certain. The study is retrospective, and the data were gathered during a period of 11 years. The treatment of cardiac arrest patients has evolved during the data gathering period and the 4-year delay from the end of follow-up to the publication of the study. Unfortunately, distinction between OHCA and IHCA, which have largely different aetiology, was not possible in the main cohort. Furthermore, the FICC database provided only a point measure of SOFA score as the worst values recorded during the first $24 \mathrm{~h}$, not allowing for evaluation of the temporal change in SOFA score.

\section{Conclusions}

We conclude that extracerebral organ failure, measured as the 24h-EC-SOFA score, is associated with long-term outcome and gross healthcare-associated costs of ICU-treated cardiac arrest patients. These associations encourage future research on the potential of EC-SOFA or SOFA score as end points in randomized controlled trials on cardiac arrest. From the clinical point of view, our data remind us that the full spectrum of multiple organ failure must be considered in the pursuit to reduce morbidity, increase survival, and optimize the use of healthcare resources after cardiac arrest.

\section{Additional files}

Additional file 1: Table S1. Characteristics of the nested cohort.
(PDF $51 \mathrm{~kb}$ )

Additional file 2: Figure S1. Distribution of EC-SOFA and SOFA score in outcome groups. (PDF $55 \mathrm{~kb}$ )

Additional file 3: Figure S2. One-year survival stratified by admission year. (PDF 38 kb)

Additional file 4: Table S2. Logistic regression model for the association of the SOFA score with outcome. (PDF $43 \mathrm{~kb}$ )

Additional file 5: Table S3. Logistic regression model for the association of the EC-SOFA sub-score with outcome in the full data. (PDF $40 \mathrm{~kb}$ )

Additional file 6: Table S4. Univariate analysis of variables used in multivariate models. (PDF $69 \mathrm{~kb}$ )

Additional file 7: Figure S3. ECPS stratified by admission year. (PDF $40 \mathrm{~kb}$ ) Additional file 8: Figure S4. Total costs per patient stratified by admission year. (PDF $46 \mathrm{~kb}$ )

Additional file 9: Table S5. Logistic regression model for the association of the EC-SOFA sub-score with outcome in the nested cohort. (PDF $40 \mathrm{~kb}$ )

Additional file 10: Table S6. Logistic regression model for the association of the EC-SOFA score with outcome in OHCA and IHCA sub-groups of the nested cohort. (PDF $40 \mathrm{~kb}$ )

Additional file 11: Table S7. Linear regression model for the association of the EC-SOFA sub-score with cost per day alive. (PDF $43 \mathrm{~kb}$ )

Additional file 12: Table S8. Linear regression model for the association of the EC-SOFA sub-score with total costs in 1-year survivors. (PDF 46 kb)

Additional file 13: Table S9. Linear regression model for the association of the EC-SOFA sub-score with cost per day alive in OHCA and IHCA sub-groups of the nested cohort. (PDF $43 \mathrm{~kb}$ )

Additional file 14: Table S10. Linear regression model for the association of the EC-SOFA sub-score with total costs in 1-year survivors in OHCA and IHCA sub-groups of the nested cohort. (PDF $43 \mathrm{~kb}$ )

\section{Abbreviations}

24h-EC-SOFA: Extracerebral sequential organ-failure assessment, worst values of the first $24 \mathrm{~h}$; Cl: Confidence interval; CNS: Central nervous system; CPC: Cerebral Performance Categories; CPI: Consumer price index; ECPS: Effective cost per survivor; EC-SOFA: Extracerebral Sequential Organ Failure Assessment (excluding the CNS sub-score); FICC: Finnish Intensive Care Consortium; ICU: Intensive care unit; IHCA: In-hospital cardiac arrest; IQR: Inter-quartile range; MOF: Multiple organ failure; OHCA: Out-of-hospital cardiac arrest; OR: Odds ratio; ROSC: Return of spontaneous circulation; SOFA: Sequential Organ Failure Assessment; WHO/ECOG: World Health Organization-Eastern Cooperative Oncology Group

\section{Funding}

Orion Research Foundation sr., Finska Läkaresällskapet, Viipurin tuberkuloosisäätiö, Svenska Kulturfonden, Medicinska Understödsföreningen Liv and Hälsa, Maud Kuistilan Säätiö, Eemil Aaltosen Säätiö, Ella and Georg Ehrnroothin Säätiö, Suomalais-Norjalainen Lääketieteen Säätiö, Suomen Lääketieteen Säätiö, Maire Taposen Säätiö, and Helsinki University Hospital (state funding: VTRTYH2014221).

\section{Availability of data and materials}

The datasets used during the current study are available from the corresponding author upon reasonable request.

\section{Authors' contributions}

PTP analysed the data and wrote the first draft of the manuscript; IE, RR, EL and MBS assisted PTP in the statistical analysis of the data; IE, RR, DF and MBS collected the healthcare cost data; MB, RL, SB, SH, TA-K, MR and MBS planned and supervised the collection of clinical data; PTP, RR, MR and MBS planned the study; PTP and MBS wrote the manuscript. All authors read and revised the manuscript text critically for important intellectual content and accepted the final version of the manuscript. 


\section{Ethics approval and consent to participate}

The study was approved by the Ethical committee of the Operative Division of Helsinki University Hospital (194/13/03/02/2014 TMK02 §97), the Finnish National Institute for Health and Welfare (THL/713/5.05.01/2014), Statistics Finland (TK-53-1047- 14), the Finnish Social Insurance Institution (Kela 55/ 522/ 2015), and the Office of the Data Protection Ombudsman (2794/204/ 2015). The need for informed consent was waived based on the retrospective non-interventional nature of the study.

\section{Consent for publication}

Not applicable.

\section{Competing interests}

The authors declare that they have no competing interests.

\section{Publisher's Note}

Springer Nature remains neutral with regard to jurisdictional claims in published maps and institutional affiliations.

\begin{abstract}
Author details
${ }^{1}$ Division of Intensive Care Medicine, Department of Anaesthesiology, Intensive Care and Pain Medicine, University of Helsinki and Helsinki University Hospital, PB 340, 00029 Helsinki, HUS, Finland. ${ }^{2}$ Department of Neurosurgery, University of Helsinki and Helsinki University Hospital, Helsinki, Finland. ${ }^{3}$ Division of Perioperative Services, Intensive Care and Pain Medicine, Turku University Hospital, Turku, Finland. ${ }^{4}$ Division of Intensive Care Medicine, Kuopio University Hospital, Kuopio, Finland. ${ }^{5}$ Department of Intensive Care, Tampere University Hospital, Tampere, Finland. ${ }^{6}$ Department of Anaesthesiology, University of Oulu, Oulu, Finland. 'Division of Intensive Care Medicine, Medical Research Center Oulu, Oulu University Hospital, Oulu, Finland. ${ }^{8}$ University of Eastern Finland and Kuopio University Hospital, Kuopio, Finland. 'Department of Emergency Care and Services, University of Helsinki and Helsinki University Hospital, Helsinki, Finland.
\end{abstract}

\section{Received: 19 November 2018 Accepted: 14 February 2019} Published online: 28 February 2019

\section{References}

1. Nolan JP, Berg RA, Callaway CW, Morrison $\sqcup$, Nadkarni V, Perkins GD, et al. The present and future of cardiac arrest care: international experts reach out to caregivers and healthcare authorities. Intensive Care Med. 2018;44:823-32.

2. Monsieurs KG, Nolan JP, Bossaert LL, Greif R, Maconochie IK, Nikolaou NI, et al. European Resuscitation Council guidelines for resuscitation 2015: section 1. Executive summary. Resuscitation. 2015;95:1-80.

3. Nolan JP, Neumar RW, Adrie C, Aibiki M, Berg RA, Bottiger BW, et al. Postcardiac arrest syndrome: epidemiology, pathophysiology, treatment, and prognostication. A scientific statement from the International Liaison Committee on Resuscitation; the American Heart Association Emergency Cardiovascular Care Committee; the Council on Cardiovascular Surgery and Anesthesia; the Council on Cardiopulmonary, Perioperative, and Critical Care; the Council on Clinical Cardiology; the Council on Stroke. Resuscitation. 2008;79:350-79.

4. Adrie C, Adib-Conquy M, Laurent I, Monchi M, Vinsonneau C, Fitting C, et al. Successful cardiopulmonary resuscitation after cardiac arrest as a "sepsislike" syndrome. Circulation. 2002;106:562-8.

5. Vincent JL, Moreno R, Takala J, Willatts S, De Mendonca A, Bruining H, et al. The SOFA (Sepsis-related Organ Failure Assessment) score to describe organ dysfunction/failure. On behalf of the Working Group on Sepsis-Related Problems of the European Society of Intensive Care Medicine. Intensive Care Med. 1996;22:707-10.

6. Ferreira FL, Bota DP, Bross A, Melot C, Vincent JL. Serial evaluation of the SOFA score to predict outcome in critically ill patients. JAMA. 2001;286:1754-8.

7. Garcia Lizana F, Peres Bota D, De Cubber M, Vincent JL. Long-term outcome in ICU patients: what about quality of life? Intensive Care Med. 2003;29: 1286-93.

8. Minne L, Abu-Hanna A, de Jonge E. Evaluation of SOFA-based models for predicting mortality in the ICU: a systematic review. Crit Care. 2008;12:R161.

9. Sakr Y, Lobo SM, Moreno RP, Gerlach H, Ranieri VM, Michalopoulos A, et al. Patterns and early evolution of organ failure in the intensive care unit and their relation to outcome. Crit Care. 2012;16:R222.
10. de Grooth HJ, Geenen IL, Girbes AR, Vincent JL, Parienti JJ, Oudemans-van Straaten HM. SOFA and mortality endpoints in randomized controlled trials: a systematic review and meta-regression analysis. Crit Care. 2017;21:38.

11. Vincent $J \mathrm{~L}$, de Mendonca A, Cantraine F, Moreno R, Takala J, Suter PM, et al. Use of the SOFA score to assess the incidence of organ dysfunction/failure in intensive care units: results of a multicenter, prospective study. Working group on "sepsis-related problems" of the European Society of Intensive Care Medicine. Crit Care Med. 1998;26:1793-800.

12. Roberts BW, Kilgannon JH, Chansky ME, Mittal N, Wooden J, Parrillo JE, et al. Multiple organ dysfunction after return of spontaneous circulation in postcardiac arrest syndrome. Crit Care Med. 2013;41: 1492-501.

13. Cour M, Bresson D, Hernu R, Argaud L. SOFA score to assess the severity of the post-cardiac arrest syndrome. Resuscitation. 2016;102:110-5.

14. Nobile L, Taccone FS, Szakmany T, Sakr Y, Jakob SM, Pellis T, et al. The impact of extracerebral organ failure on outcome of patients after cardiac arrest: an observational study from the ICON database. Crit Care. 2016;20:368.

15. Reinikainen $M$, Oksanen $T$, Leppanen $P$, Torppa T, Niskanen M, Kurola J, et al Mortality in out-of-hospital cardiac arrest patients has decreased in the era of therapeutic hypothermia. Acta Anaesthesiol Scand. 2012;56:110-5.

16. Reinikainen M, Mussalo P, Hovilehto S, Uusaro A, Varpula T, Kari A, et al. Association of automated data collection and data completeness with outcomes of intensive care. A new customised model for outcome prediction. Acta Anaesthesiol Scand. 2012;56:1114-22.

17. Oken MM, Creech RH, Tormey DC, Horton J, Davis TE, McFadden ET, et al. Toxicity and response criteria of the Eastern Cooperative Oncology Group. Am J Clin Oncol. 1982;5:649-55.

18. Efendijev I, Folger D, Raj R, Reinikainen M, Pekkarinen PT, Litonius E, et al. Outcomes and healthcare-associated costs one year after intensive caretreated cardiac arrest. Resuscitation. 2018;131:128-34.

19. Cummins RO, Chamberlain DA, Abramson NS, Allen M, Baskett PJ, Becker $L$, et al. Recommended guidelines for uniform reporting of data from out-of-hospital cardiac arrest: the Utstein style. A statement for health professionals from a task force of the American Heart Association, the European Resuscitation Council, the Heart and Stroke Foundation of Canada, and the Australian Resuscitation Council. Circulation. 1991;84:960-75.

20. Perkins GD, Jacobs IG, Nadkarni VM, Berg RA, Bhanji F, Biarent D, et al. Cardiac arrest and cardiopulmonary resuscitation outcome reports: update of the Utstein resuscitation registry templates for out-ofhospital cardiac arrest: a statement for healthcare professionals from a task force of the International Liaison Committee on Resuscitation (American Heart Association, European Resuscitation Council, Australian and New Zealand Council on Resuscitation, Heart and Stroke Foundation of Canada, InterAmerican Heart Foundation, Resuscitation Council of Southern Africa, Resuscitation Council of Asia); and the American Heart Association Emergency Cardiovascular Care Committee and the Council on Cardiopulmonary, Critical Care, Perioperative and Resuscitation. Resuscitation. 2015;96:328-40.

21. Jennett $B$, Bond M. Assessment of outcome after severe brain damage. Lancet (London, England). 1975;1:480-4.

22. Group BRCTIS. A randomized clinical study of cardiopulmonary-cerebral resuscitation: design, methods, and patient characteristics. Am J Emerg Med. 1986:4:72-86.

23. STROBE-statement. http://www.strobe-statement.org. Accessed 9 Jan 2019.

24. Yoon JC, Kim YJ, Lee YJ, Ryoo SM, Sohn CH, Seo DW, et al. Serial evaluation of SOFA and APACHE II scores to predict neurologic outcomes of out-ofhospital cardiac arrest survivors with targeted temperature management. PLoS One. 2018;13:e0195628.

25. Choi JY, Jang JH, Lim YS, Jang JY, Lee G, Yang HJ, et al. Performance on the APACHE II, SAPS II, SOFA and the OHCA score of post-cardiac arrest patients treated with therapeutic hypothermia. PLoS One. 2018;13:e0196197.

26. Stanger DE, Fordyce CB. The cost of care for cardiac arrest. Resuscitation. 2018;131:A7-8.

27. Jakobsson J, Nyquist O, Rehnqvist N, Norberg KA. Cost of a saved life following out-of-hospital cardiac arrest resuscitated by specially trained ambulance personnel. Acta Anaesthesiol Scand. 1987;31:426-9.

28. Graf J, Muhlhoff C, Doig GS, Reinartz S, Bode K, Dujardin R, et al. Health care costs, long-term survival, and quality of life following intensive care unit admission after cardiac arrest. Crit Care. 2008;12:R92. 
29. Naess AC, Steen PA. Long term survival and costs per life year gained after out-of-hospital cardiac arrest. Resuscitation. 2004;60:57-64.

30. Petrie J, Easton S, Naik V, Lockie C, Brett SJ, Stumpfle R. Hospital costs of out-ofhospital cardiac arrest patients treated in intensive care; a single centre evaluation using the national tariff-based system. BMJ Open. 2015;5:e005797.

31. van Alem AP, Dijkgraaf MG, Tijssen JG, Koster RW. Health system costs of out-of-hospital cardiac arrest in relation to time to shock. Circulation. 2004 110:1967-73.

Ready to submit your research? Choose BMC and benefit from:

- fast, convenient online submission

- thorough peer review by experienced researchers in your field

- rapid publication on acceptance

- support for research data, including large and complex data types

- gold Open Access which fosters wider collaboration and increased citations

- maximum visibility for your research: over $100 \mathrm{M}$ website views per year

At $\mathrm{BMC}$, research is always in progress.

Learn more biomedcentral.com/submissions 